\title{
Stabilizing forces acting on ZnO polar surfaces: STM, LEED, and DFT
}

\author{
H. Xu, ${ }^{1}$ L. Dong, ${ }^{2}$ X. Q. Shi, ${ }^{1}$ M. A. Van Hove,${ }^{3}$ W. K. Ho, ${ }^{4}$ N. Lin, ${ }^{2}$ H. S. Wu, ${ }^{4}$ and S. Y. Tong ${ }^{1, *}$ \\ ${ }^{1}$ Department of Physics, South University of Science and Technology of China, China \\ ${ }^{2}$ Department of Physics, The Hong Kong University of Science and Technology, Clear Water Bay, Hong Kong, China \\ ${ }^{3}$ Institute of Computational and Theoretical Studies and Department of Physics, Hong Kong Baptist University, Hong Kong, China \\ ${ }^{4}$ Department of Physics, The University of Hong Kong, Pokfulam Road, Hong Kong, China \\ (Received 16 July 2012; revised manuscript received 25 March 2014; published 5 June 2014)
}

\begin{abstract}
New understanding has been reached on competing forces acting to stabilize the polar surfaces of intrinsic $\mathrm{ZnO}$. To compensate an accumulating dipole moment normal to ionic planes, the Madelung electrostatic force and the bonding ability of undercoordinated $\mathrm{Zn}$ ions compete to deplete more $\mathrm{Zn}$ atoms from the (0001) face and more $\mathrm{O}$ atoms from the $(\mathbf{0 0 0} \overline{\mathbf{1}})$ face. In this competition, the former mechanism wins because it provides very low energy binding sites for $\mathrm{O}$ ions at face-centered-cubic registries on both surfaces. On the $\mathrm{Zn}$-face, a distorted tetrahedral structure is formed, while on the O-face, a vertical Y structure is formed. In both structures, $\mathrm{O}$ ions form the topmost atomic plane. The reconstructed polar surfaces containing these novel structures have cleavage energy of $2.36 \mathrm{~J} / \mathrm{m}^{2}$, comparable to that of nonpolar surfaces and in agreement with experimental observation. An earlier structure found on the $\mathrm{Zn}$-face annealed at below $1000 \mathrm{~K}$ is stabilized mainly by the Madelung electrostatic force and is a metastable structure of that surface.
\end{abstract}

DOI: 10.1103/PhysRevB.89.235403

PACS number(s): 68.35.B-, 68.37.Ef, 68.43.Bc, 68.47.Gh

\section{INTRODUCTION}

The Zn-terminated (0001) face and O-terminated (000) $\overline{1})$ face (hereafter referred to as $\mathrm{Zn}$-face and $\mathrm{O}$-face, respectively) of $\mathrm{ZnO}$ have received widespread attention because these surfaces are active in catalytic and corrosion reactions. Also, $\mathrm{ZnO}$ nanorods and nanowires grown along the $c$ axis, bounded at their ends by these polar faces, have exhibited remarkable functional properties $[1,2]$. The active roles played by polar faces make understanding their structure and stabilizing mechanism a high priority. Previous works [3-5] have noted that a stack of ionic (0001) planes of opposite charges sets up a net dipole field that accumulates along the crystal's $c$ axis. To obtain finite surface energies for the polar faces of a thick $\mathrm{ZnO}$ slab [3,4], a counter field has to be present to cancel the dipole moment set up by the ionic planes. One way to do this is to deposit extra electrons on the Zn surface and extra "holes" (electrons are taken away) on the O-face. The surface structure may remain $(1 \times 1)$ bulklike. In Sec. III, we shall discuss this situation in more detail. A competing stabilizing mechanism is for the surfaces to undergo stoichiometric change and surface rebonding. One proposed reconstruction is to have approximately $25 \%$ more $\mathrm{Zn}$ atoms than $\mathrm{O}$ atoms removed from the $\mathrm{Zn}$-face and vice versa from the $\mathrm{O}$-face. The purpose of the "differential removal" mechanism is twofold: (1) it compensates the macroscopic dipole field without cumulating extra electrons at the $\mathrm{Zn}$-face or extra holes at the O-face, and (2) through rebinding, it creates new structures whose energies are lower than that of the threefold coordinated $\mathrm{Zn}$ ions or $\mathrm{O}$ ions found on $1 \times 1$ unreconstructed surfaces. Two widely cited studies [6,7] have supported the differential removal of surface ions from the two polar faces as the major stabilizing process. On the Zn-face, Dulub et al. [6] suggested that surface cavities of various sizes are formed by removing $\mathrm{Zn}$ and $\mathrm{O}$ atoms from the surface bilayer. To form a triangular cavity of

*Corresponding author: tong.sy@ sustc.edu.cn single-bilayer depth with side $n \mathrm{Zn}$ atoms, $n(n+1) / 2 \mathrm{Zn}$ atoms and $n(n-1) / 2 \mathrm{O}$ atoms are removed, resulting in removing $n^{2}$ atoms with a net removal of $n \mathrm{Zn}$ atoms per triangular cavity. The driving force for this model is the electrostatic Madelung force [8]; however, this model fails to explain the O-face. Lauritsen et al. [7] have shown that although surface cavities of various sizes are also found on the O-face, the surface reconstruction on the $\mathrm{O}$-face is driven by the bonding flexibility of undercoordinated (threefold bonded) $\mathrm{Zn}$ ions in the first bilayer. The need for entirely different driving forces to explain the reconstructions on the two polar faces is puzzling because the top layer of an unreconstructed $\mathrm{Zn}$-face is entirely made up of undercoordinated $\mathrm{Zn}$ ions; hence, the bonding flexibility effect proposed for the O-face should also be present on the Zn-face. Similarly, the electrostatic Madelung force proposed to drive the reconstruction on the $\mathrm{Zn}$-face should also be present on the O-face. Thus, a definitive statement on reconstruction models of $\mathrm{ZnO}$ polar surfaces is still missing.

In this paper, we present evidence, based on lowtemperature scanning tunneling microscopy (STM), dynamical low energy electron diffraction (LEED), and density functional theory (DFT) calculations that shows a unified picture exists to explain the stabilization of both polar surfaces. We show that, unlike the conclusions given in previous works [6,7], the bonding flexibility of undercoordinated $\mathrm{Zn}$ is responsible for the most stable reconstruction structures found on both the $\mathrm{Zn}$ - and O-faces. We present these most stable reconstruction structures for each polar face: they are the adatom-cavity (ADC) structure on the $\mathrm{Zn}$-face and the disordered Y (DY) structure on the O-face, respectively. We also calculate the cleavage energy for polar surfaces of thick slabs containing the (ADC + DY) structures and show that it is comparable to that of nonpolar surfaces.

\section{EXPERIMENTAL AND THEORETICAL CONDITIONS}

The STM experiments were performed in a commercial ultrahigh vacuum low-temperature system (Omicron 
Nanotechnology) with base pressure $1.0 \times 10^{-10}$ mbar. All the single crystal samples, from Tokyo Denpa and Cermet, were treated in situ by Ar-ion sputtering and annealing of direct-current heating, and the cleanliness was carefully monitored by Auger electron spectroscopy that records only $\mathrm{O}$ and $\mathrm{Zn}$ peaks, indicating no detectable impurities present on the surface. Different annealing temperatures were employed for the Zn-face, $900 \mathrm{~K}-1300 \mathrm{~K}$, and for the O-face, $850 \mathrm{~K}-1000 \mathrm{~K}$. STM measurements were carried out at $5 \mathrm{~K}$ or $77 \mathrm{~K}$ (as indicated in the figure captions) with an etched W tip in constant-current mode, and the bias voltage was referenced to the sample. As the O-face is poorly conducting, no STM scanning at $5 \mathrm{~K}$ was performed on it. For normal incidence LEED intensity-voltage (IV) measurements, the data for the $\mathrm{Zn}$-face were collected at $140 \mathrm{~K}$, while those for the O-face were collected at room temperature, again due to the less conducting nature of the latter surface. The temperature was monitored by a K-type thermocouple located near the sample.

For the calculations, we used DFT as implemented in the Vienna $A b$ initio Simulation Package (VASP) [9] with projector-augmented wave (PAW) potentials [10] and Perdew and Wang's 1991 (PW91) functional [11]. The cutoff energy, the vacuum region, and the force convergence criterion on each atom were $450 \mathrm{eV}, 12 \AA$, and $0.02 \mathrm{eV} / \AA$, respectively. For the $\mathrm{Zn}$-face, the climbing image nudged elastic band (CI-NEB) method [12] was used to estimate potential energies of $\mathrm{Zn}$ or $\mathrm{O}$ atoms on the surface. The symmetrized automated tensor LEED computer code SATLEED $[13,14]$ was used for structural analysis. For the $\mathrm{Zn}$-face, the cumulative energy range of LEED beams is $720 \mathrm{eV}$. The widest energy range for one beam spans from 70 to $450 \mathrm{eV}$. For the O-face, the cumulative energy range of LEED beams is $650 \mathrm{eV}$. The widest energy range for one beam spans from $35 \mathrm{eV}$ to $300 \mathrm{eV}$. The agreement between the experiment and theory was quantified by the Pendry $R$-factor [15].

\section{AUTOCHARGE COMPENSATION FOR POLAR SURFACES}

Tasker [5] has classified a $\mathrm{ZnO}$ slab bounded by polar faces of opposite charges as a type III ionic structure and concluded that such a structure would have a dipole moment that diverges with thickness. Goniakowski et al. [4] have shown that above a critical thickness, the dipole moment set up by the ionic planes must be autocompensated. A closer look at this autocompensation mechanism reveals rather interesting observations. A thick $\mathrm{ZnO}$ crystalline slab has the wurtzite structure with each $\mathrm{Zn}$ ion bonded to four $\mathrm{O}$ ions and vice versa. For simplicity of argument, we assume each $\mathrm{Zn}$ to donate $-0.5 e^{*}$ to its $\mathrm{O}$ neighbor, where $e^{*}=\gamma e, \gamma$ is the fractional ionicity, and $e$ is the absolute charge of an electron. In the bulk, each $\mathrm{Zn}$ ion carries a net charge of $+2.0 e^{*}$, while each $\mathrm{O}$ ion carries a $-2.0 e^{*}$ charge. To form a slab bounded by polar faces, bulk bonds that connect a $\mathrm{Zn}$ ion to an $\mathrm{O}$ ion must be cleaved. As Fig. 1(a) shows, a cleave perpendicular to the [0001] direction always produces a $\mathrm{Zn}$-face together with an $\mathrm{O}$-face, assuming all surface ions are threefold coordinated. At the moment of cleaving, $-0.5 e^{*}$ hops from the $\mathrm{O}$ ion to the $\mathrm{Zn}$ ion, resulting in a cleaved threefold bonded $\mathrm{Zn}$ ion having a reduced net charge of $-1.5 e^{*}$ while a cleaved threefold bonded $\mathrm{O}$ having a reduced net charge of $+1.5 e^{*}$. The situation is shown in the inset of Fig. 1(a). This charge hopping at cleavage is very fast, scaling as $c / \alpha$, where $c$ is the velocity of light and $\alpha=137$ is the universal constant. The charge-hopping process occurs whether or not a slab is $100 \%$ insulating or mildly conducting (due to bulk defects) because it prevents a cleaved bond on a cation from being (electron) empty and a cleaved bond on an anion from being (electron) filled. A "dangling" cation bond filled with electrons is lower in energy than that of an empty "dangling" cation bond and vice versa for the "dangling" anion bond. After charge hopping, the slab is autocharge compensated, as shown in Fig. 1(b), and it may be
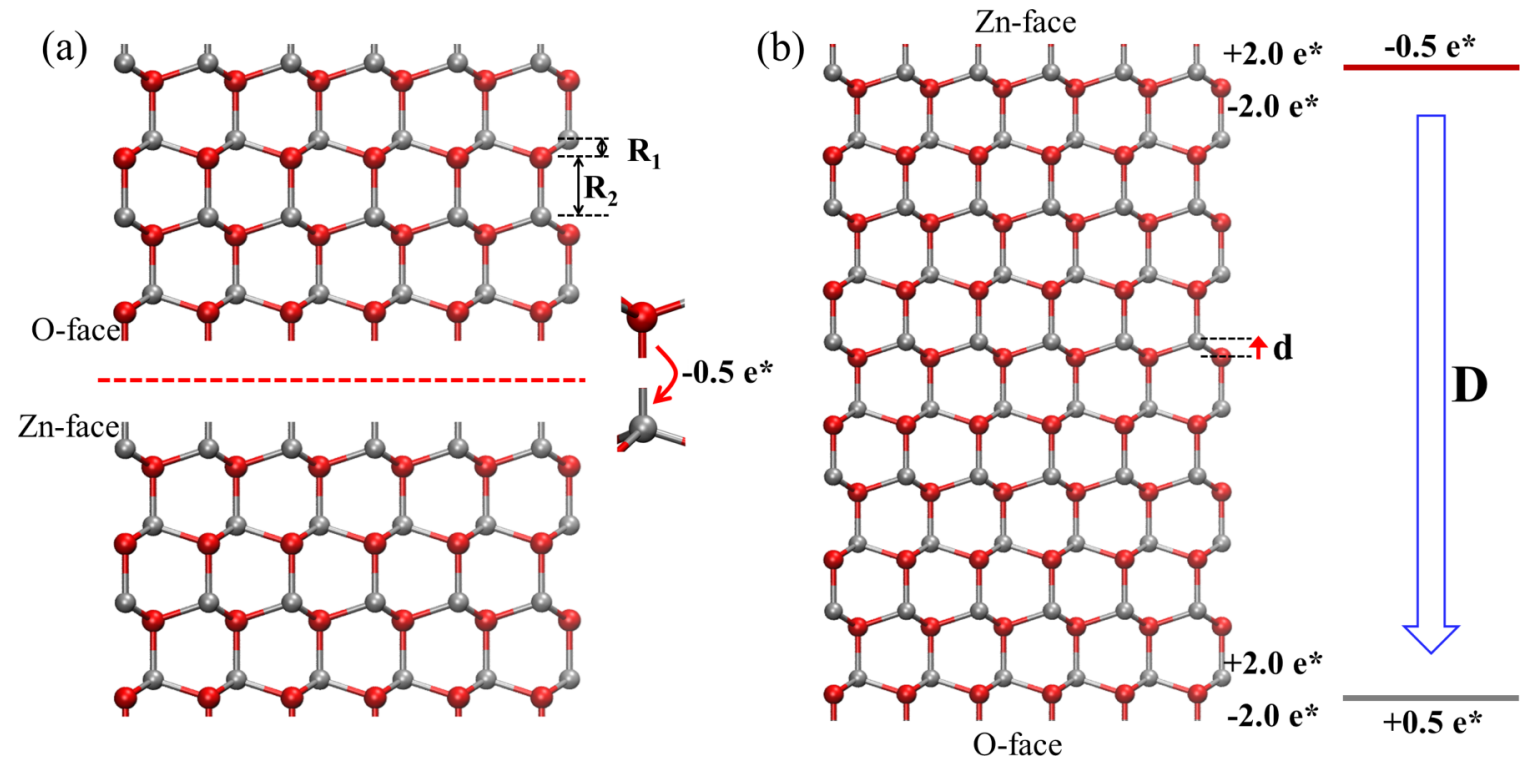

FIG. 1. (Color online) (a) Cleave (dotted line) of $\mathrm{ZnO}$ along the (0001) plane cutting single bonds. Inset: Charge hopping of $-0.5 e^{*}$ from $\mathrm{O}$ ion to $\mathrm{Zn}$ ion at moment of cleaving. (b) Left: Tasker type III ionic slab with cumulative dipole moments $d$. Right: Sheets of extra charge setting up a compensating dipole $D$. 
regarded as a Tasker type III ionic slab plus a sheet of $-0.5 e^{*}$ centered somewhere at the $\mathrm{Zn}$ surface and $+0.5 e^{*}$ centered somewhere at the $\mathrm{O}$ surface. Such an autocharge compensated slab will have a nondivergent dipole field within the slab.

It is worth mentioning that in a $(1 \times 1)$ ionic slab bounded by polar surfaces, the extra electron buildup at the Zn-face (and "hole" buildup at the O-face) is caused by the dipole moment set up by the sheets of $\mathrm{Zn}$ and $\mathrm{O}$ ions. The extra electrons at the $\mathrm{Zn}$-face will not drain to the $\mathrm{O}$-face even in conducting $\mathrm{ZnO}$ slabs (due to bulk defects) as long as the dipole moment set up by the ionic planes remains present. In a real $\mathrm{ZnO}$ slab bounded by polar surfaces, one has to replace the extra charge $\pm 0.5 e^{*}$ by $\pm 2 R_{1} /\left(R_{1}+R_{2}\right) e^{*}$, where $R_{1}$ is the bulk bilayer separation and $R_{2}$ is the nearest distance between like-ion planes. Surface and bulk lattice strain may require additional charge redistribution between the $\mathrm{Zn}$ - and O-faces, and this can be achieved via charge flow from one end of a slab to the other in mildly conducting slabs (due to bulk defects) or via discharging to the environment in perfectly insulating slabs.

The autocharge compensated slab shown in Fig. 1(b) has a $(1 \times 1)$ structure on both polar faces. Although such a slab may have a finite dipole moment, its surface energy at each end may not be the lowest because the surface regions have to accommodate extra charge. As we show in Sec. IV, for $\mathrm{ZnO}$ the $(1 \times 1)$ structure is unstable on either polar surface for thick slabs. The cleavage energy of a thick $\mathrm{ZnO}$ slab bounded by $(1 \times 1)$ polar surfaces is more than $40 \%$ higher than that bounded by the most stable reconstructed surfaces. The purpose of this work is to find the most stable reconstructed structures on both polar faces.

\section{THE ADC RECONSTRUCTION MODEL ON THE Zn-FACE}

An alternative to $(1 \times 1)$ surfaces is to have the surfaces undergo stoichiometric change and/or rebonding so that dipole compensation may be achieved by differential depletion of surface ions [3-8] instead of by extra charge buildup. We shall first present our results on the $\mathrm{Zn}$-face and comment on how these results differ from that of previous works $[6,8]$. Figures 2(a)-2(c) show STM images at various magnifications of the surface after the sample is annealed at $900 \mathrm{~K}$. The surface morphology consists of multi-tier triangular islands, of which the height differences are multiples of half a bulk unit cell, which is $c / 2, \sim 0.26 \mathrm{~nm}$. Figures 2(e)-2(f) show STM images at various scales of the surface after annealing at $1300 \mathrm{~K}$. The higher temperature annealing causes the dissolution of the islands to form broad terraces bounded alternately by smooth and jagged step edges, typical of the wurtzite step-flow growth mechanism [16]. The height difference between a smoothedged terrace and a jagged-edged terrace below it is $c / 2$ as well.

The most interesting features on both the $900 \mathrm{~K}$ and $1300 \mathrm{~K}$ annealed surfaces are atomic-sized spots ("eyes") populating the surface [e.g., inside red dashed circles of Figs. 2(b), 2(c), and 2(e)]. A line scan of the eyes [Fig. 2(e), inset] indicates that their planar width is approximately $0.45 \mathrm{~nm}$ from dip to dip. Besides the appearance of the eyes, cavities of various shapes and sizes populate the island and terrace surfaces. From high resolution images, it is possible to identify cavities having a single missing $\mathrm{Zn}$ atom [Fig. 2(f), top], three missing $\mathrm{Zn}$ atoms and one missing $\mathrm{O}$ atom [Fig. 2(e), circled and 2(f), middle], or six missing $\mathrm{Zn}$ atoms and three missing $\mathrm{O}$ atoms [Fig. 2(f), bottom]; larger cavities with irregular shapes are also found. Dulub et al. [6] have studied the $900 \mathrm{~K}$ surface with STM and analyzed the characteristics of the cavities, but they never reported seeing the eyes. To best image the eyes, a number of factors are important. (1) It is desirable to reduce thermal drift; hence, low-temperature scanning is definitely an advantage. (2) Annealing samples at below $1000 \mathrm{~K}$, the temperature used in Ref. [6], the surface is rough and is populated by triangular islands with small terrace areas in between. In this environment, there is a higher chance for $\mathrm{O}$ atoms to migrate to step edges and form bonds there [6] and not be captured at face-centered-cubic (fcc) sites on terraces. When the surface is annealed to $1300 \mathrm{~K}$, the higher temperature annealing causes the dissolution of the islands to form broad terraces and many more $\mathrm{O}$ atoms are trapped at fcc sites on the broad terraces. This is why many more eyes per surface area were observed on samples annealed at $1300 \mathrm{~K}$ than at $900 \mathrm{~K}$ [compare Fig. 2(e) vs 2(b)]. (3) The bias voltage is important in imaging the eyes. According to our experience, the eyes could be seen clearly at about $+2.0 \mathrm{~V}$ when the tip is sharp. If the tip is not sharp enough, lowering the bias voltage to about $+0.6 \mathrm{~V}$ would make it possible to reveal the eyes again.

To determine the elemental identity of the eyes, we recall that Auger measurements have found no trace of impurity ions on the surface. With the abundance of eyes imaged by STM, if they were due to impurity elements, Auger measurements would no doubt detect them. Also, the annealing temperature is high enough to completely dehydroxylate the surface, and we detected no correlation between the number of eyes imaged and scanning time, thus ruling out any re-adsorption effect of hydrogen. This means the eyes can only be atoms of $\mathrm{Zn}$ or $\mathrm{O}$ adsorbed on the surface. We have used DFT to calculate potential energy profiles for surface $\mathrm{Zn}$ and $\mathrm{O}$ atoms located at different positions on the surface The DFT calculations use a $(6 \times 6)$ unit cell and seven bilayers, and we stabilize the O-face of the slab with pseudohydrogen atoms (valence 0.5 ) placed at on-top sites of a bulklike $\mathrm{O}$ surface, and structural relaxations are allowed for all atoms in the slab. The potential energy profile in Fig. 3(a) shows that a $\mathrm{Zn}$ atom leaving a lattice site on the surface must encounter an initial energy barrier of about $0.80 \mathrm{eV}$. Afterward, the potential energy profile of the $\mathrm{Zn}$ atom on the surface stays very flat. This is to be expected because the $\mathrm{Zn}$ atom is unlikely to form ionic bonds with the underlying $\mathrm{Zn}$ surface. The situation is quite different for a surface $\mathrm{O}$ atom leaving a lattice site next to a $\mathrm{Zn}$ vacancy. As shown in the potential energy profile of Fig. 3(b), after an initial barrier of $0.60 \mathrm{eV}$, a number of local minima exists on the surface to "trap" the $\mathrm{O}$ atom; the most stable trap is at a fcc site at $0.86 \mathrm{~nm}$. Compared to its original lattice site next to a $\mathrm{Zn}$ vacancy, each $\mathrm{O}$ trapped at the fcc site gains $\sim 0.53 \mathrm{eV}$ in binding energy. These results indicate that while the $\mathrm{Zn}$ atom is free to migrate long distances on the surface, the $\mathrm{O}$ atoms have a high probability of being recaptured at fcc sites. We name the new structure containing such fcc-bonded $\mathrm{O}$ adatoms the ADC model. It is many times more efficient in differentially depleting surface $\mathrm{Zn}$ atoms than the cavity model [6] - by a factor of $(n+1) / 2$, where $n$ is the number of missing $\mathrm{Zn}$ 
(a)

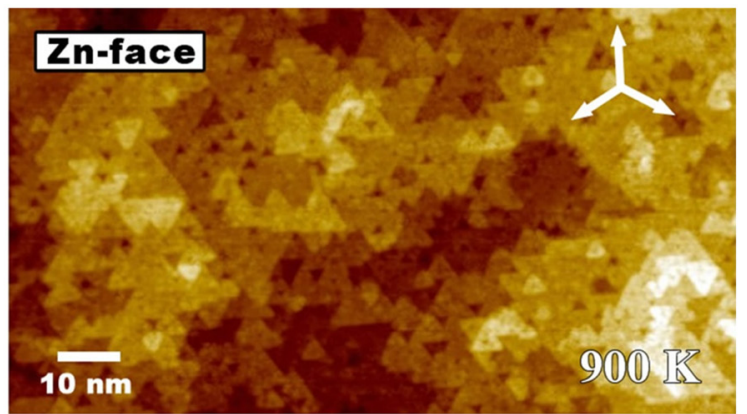

(b)

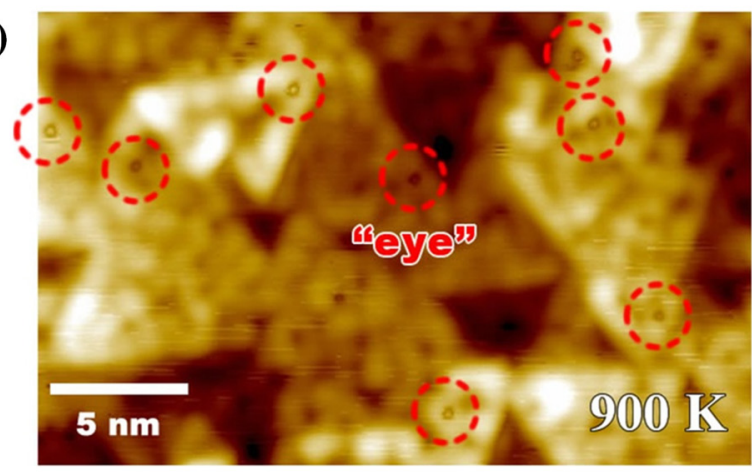

(c)

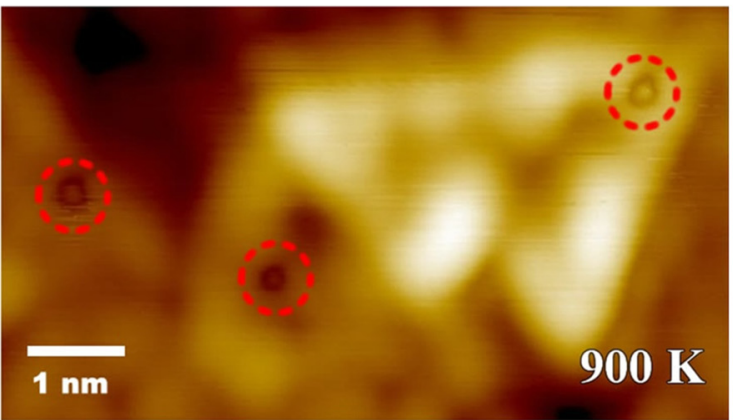

(d)

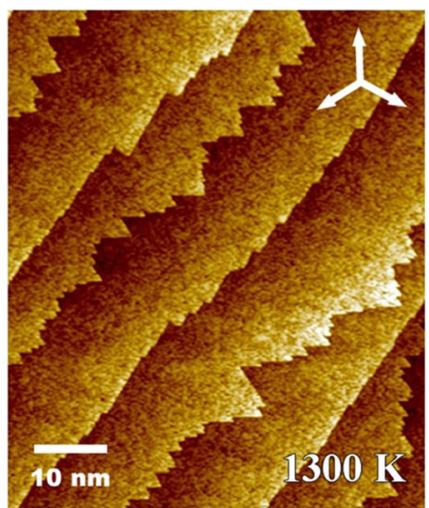

(e)

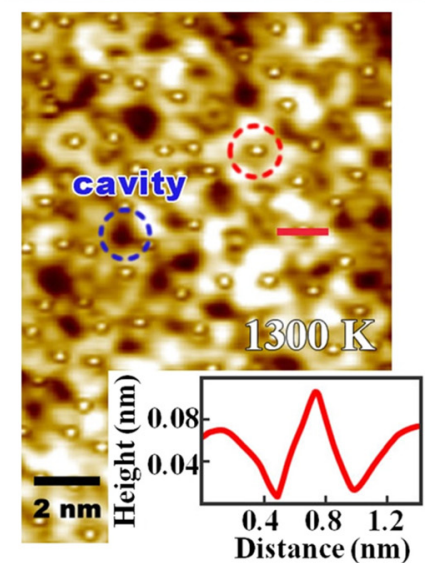

(f)

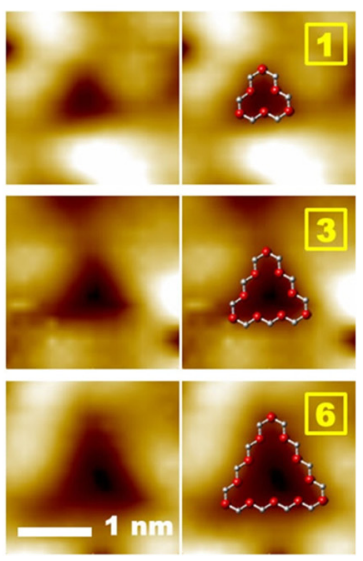

FIG. 2. (Color online) (a) STM image of the Zn-face after annealing at $900 \mathrm{~K}$. (b), (c) Magnified views of the islands in (a). (d) Large scale STM image of the Zn-face after annealing at $1300 \mathrm{~K}$. (e) Zoom into a terrace after $1300 \mathrm{~K}$ annealing, with an eye and a cavity marked by red and blue dashed circles, respectively. The insert shows the height profile of the STM image along the red horizontal line. (f) Cavities of different sizes (left) overlaid with atomic models (right: $\mathrm{O}$, red; $\mathrm{Zn}$, gray); the labels enclosed in squares indicate the number of missing $\mathrm{Zn}$ ions. The cavity circled in (e) is of type III in (f). All the images were obtained at $77 \mathrm{~K}$ except (f) at $5 \mathrm{~K}$. The bias voltage is $+3.0 \mathrm{~V}$ in (a) and $+2.0 \mathrm{~V}$ in (b)-(f).

atoms per side of a triangular cavity. Compared to the cavity model [6], the ADC model achieves the required quenching of macroscopic dipole field by forming far fewer cavities on the surface.

To compare energies of the different models, we used DFT and calculated the total energies of four reconstruction models on the Zn-polar face. The four models are, respectively, referring to Fig. 4(a), $(2 \times 2)$ single $\mathrm{Zn}$ vacancies $(\mathrm{Va})$; Fig. 4(b), $(2 \times 2) \mathrm{O}$ adatoms at fcc hollow sites (Ad); Fig. 4(c), the cavity model [6] (Ca); and Fig. 4(d), the ADC model. In each model, there is either a net loss of $25 \%$ surface $\mathrm{Zn}$ atoms [models (a), (c) and (d)] or net gain of $25 \%$ surface $\mathrm{O}$ atoms [model (b)]. The DFT calculations use a $(6 \times 6)$ unit cell and seven bilayers. For the slab calculation, we stabilize the O-face with pseudohydrogen atoms (valence 0.5 ) placed at on-top sites of a bulklike $\mathrm{O}$ surface. Structural relaxations are allowed for all atoms in the slab. The total energies of the four models are shown in Fig. 4(e), which shows that the ADC model has by far the lowest energy by more than $1.00 \mathrm{eV}$ per $(6 \times 6)$ unit cell compared to that of the cavity model [6] and more than $1.23 \mathrm{eV}$ per unit cell compared to that of the ordered single $\mathrm{Zn}$ vacancy model. 


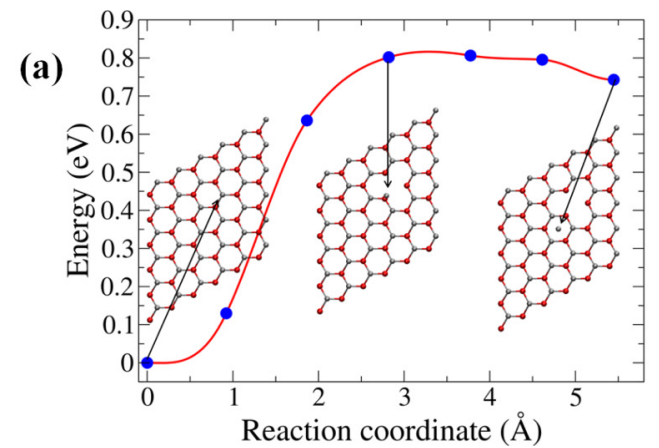

(b)

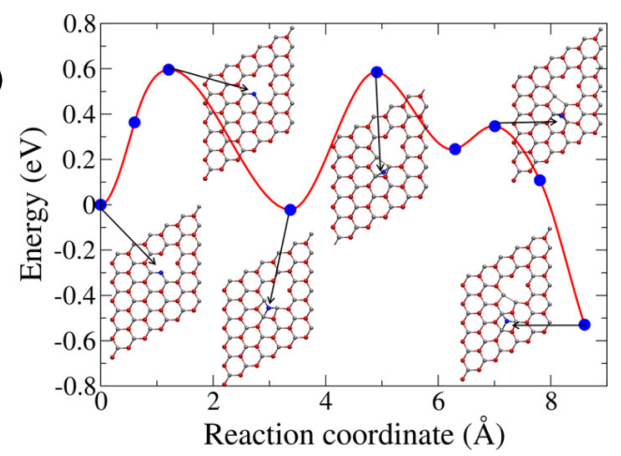

FIG. 3. (Color online) (a) Potential energy profile for a surface $\mathrm{Zn}$ atom leaving a lattice site (leftmost data point) on the $\mathrm{Zn}$-face and at different locations of the surface (blue dots). (b) Reaction barriers for an $\mathrm{O}$ atom leaving a lattice site next to a $\mathrm{Zn}$ vacancy and at different locations of the surface.

To further support the validity of the ADC model on the $\mathrm{Zn}$-face, we have carried out dynamical LEED IV spectra analysis to determine the reconstruction of the $\mathrm{Zn}$-face. The LEED IV curves were measured on the sample annealed at $1300 \mathrm{~K}$. The pattern exhibits $(1 \times 1)$ spots with diffuse background due to the disorder seen by STM. The symmetry is $\mathrm{C}_{3 \mathrm{v}}$ for the ideal bulk-terminated surface, while the LEED pattern exhibits sixfold symmetry due to equal areas of exposed
A- and B-type terraces of the wurtzite structure [16]. In terms of surface structure, the exposed A- and B-type terraces are rotated $180^{\circ}$ from each other. Due to the high symmetry of the LEED pattern, only three symmetrically inequivalent beams could be measured at normal incidence. We compared the calculated intensities of integer-order beams [light lines in Fig. 5(a)] with those of the measured beams [bold lines in Fig. 5(a)], on the assumption that the spot intensities are
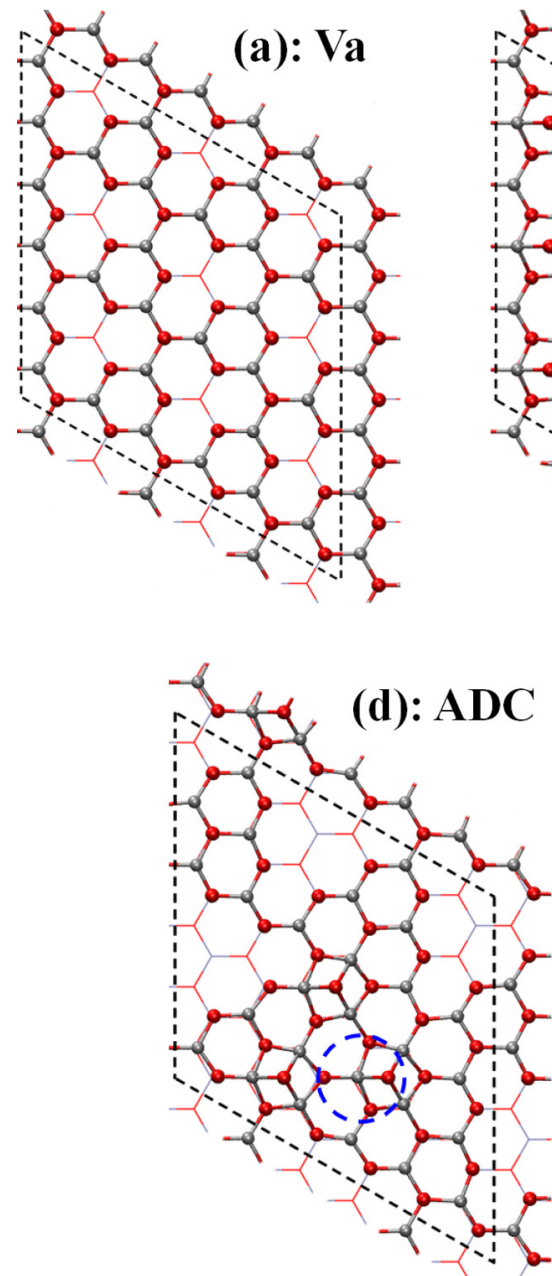
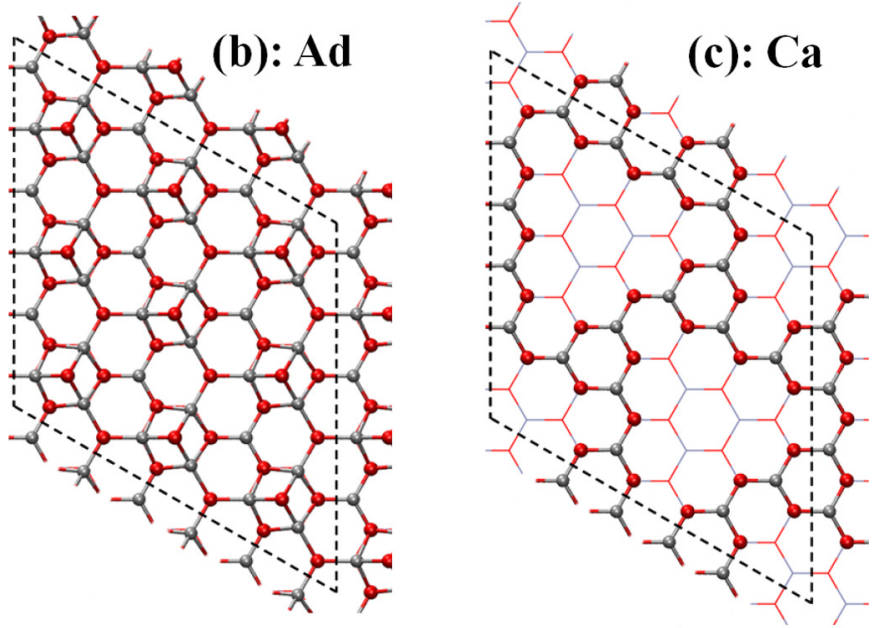

FIG. 4. (Color online) (a)-(d) Top views of reconstructions on the $\mathrm{Zn}$-face in a $(6 \times 6)$ unit cell for models: Va, Ad, Ca, and ADC. (e) Calculated total energies of the respective models, with the total energy of model Va set to zero. 
(a)

(b)

\begin{tabular}{|c|c|}
\hline Surface model & Pendry R-factors \\
\hline Unreconstructed: $(1 \times 1)$ cell & 0.48 \\
\hline Zn vacancy: $(2 \times 2)$ cell & 0.40 \\
\hline O-adatom: $(2 \times 2)$ cell & 0.34 \\
\hline Cavity model: $(4 \times 4)$ cell & 0.33 \\
\hline $\begin{array}{c}\text { Adatom-cavity }(\text { ADC): } \\
(2 \sqrt{ } 3 \times 2 \sqrt{ } 3) \text { R } 30^{\circ} \text { cell }\end{array}$ & 0.24 \\
\hline
\end{tabular}

FIG. 5. (a) Experimental (bold lines) and calculated (light lines) LEED IV curves for the ADC model. Individual beam $R$-factors are given. (b) The different tested models with the respective Pendry $R$-factors. The boundaries of unit cells are shown as dashed lines. O: red spheres, $\mathrm{Zn}$ : gray spheres.

relatively insensitive to the disorder (which mainly diffracts in directions between beams). Prior work comparing LEED and diffuse LEED IV spectra has established the validity of this approach $[17,18]$. A number of structural models were tested, and the respective Pendry $R$-factors are listed in Fig. 5(b). The results confirm the validity of the ADC model, with a Pendry $R$-factor of 0.24 that is $35 \%$ smaller (i.e., better) than that of the next best model (the cavity model [6]).

\section{THE DY RECONSTRUCTION MODEL ON THE O-FACE}

Turning to the O-face, previous $\mathrm{x}$-ray photoelectron spectroscopy $[19,20]$ and thermal desorption spectroscopy studies

(a)

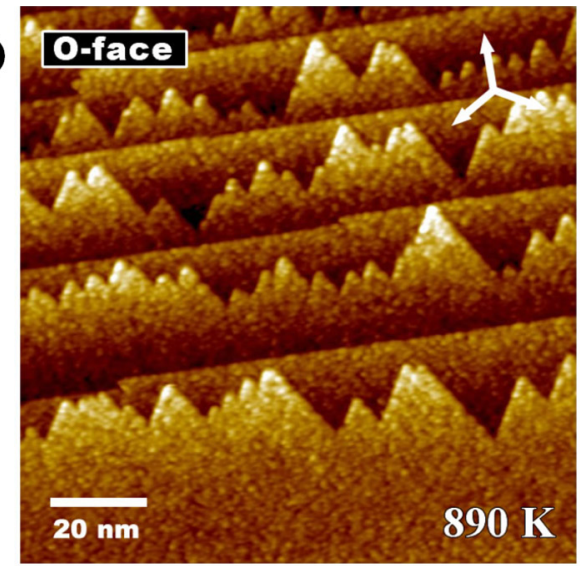

[21] have established that a completely hydrogen free $\mathrm{O}$ surface can be obtained by annealing the sample at temperatures in excess of $500^{\circ} \mathrm{C}$. As we wish to study the intrinsic reconstruction structure of the O-face, both samples are annealed above this temperature. Figure 6(a) shows an image of the cermet sample annealed at $890 \mathrm{~K}$, revealing layers of alternating jagged- and smooth-edged terraces separated by single bilayer $c / 2$ heights, similar to what was observed on the $\mathrm{Zn}$ polar face. High-resolution STM images of areas on terraces reveal a surface morphology with sub-nm roughness having two distinct features. A representative image, shown in Fig. 6(b), shows the following. (1) The surface is populated by disordered pits, as marked by the arrows. The pits have irregular shapes and are quite deep. (2) In regions between the pits, the surface shows a roughness of about $0.05 \mathrm{~nm}$, as shown by the cross-section profile [Fig. 6(c)]. Lauritsen et al. [7] have observed ordered $(5 \times 5)$ pits on samples annealed at $450{ }^{\circ} \mathrm{C}$, an annealing temperature below complete dehydroxylation. Their structural model also contains four $\mathrm{OH}$ groups per $(5 \times 5)$ unit cell [7]. Such ordered $(5 \times 5)$ pits are not observed on our samples presumably because all surface hydrogen has been driven off by the higher annealing temperature. Careful monitoring of the STM images as a function of scanning time revealed no apparent increase in surface ordering, indicating hydrogen readsorption during scanning is minimal.

With insight gained from the $\mathrm{Zn}$-face regarding the interplay of the Madelung force and bonding flexibility of undercoordinated $\mathrm{Zn}$ ions, we introduce a new model where the top $\mathrm{O}$ ions occupy both fcc and hexagonal close-packed (hcp) sites and the $\mathrm{Zn}$ ion bonded to these $\mathrm{O}$ ions is threefold coordinated, forming a vertical Y [perpendicular to the (0001) plane] structure with the fcc and hep $\mathrm{O}$ ions above and an $\mathrm{O}$ ion directly below in the next bilayer. Two vertical Y structures are present in the new model, shown in Fig. 7(e): a planar Y structure marked by the red dashed ellipse and a distorted $\mathrm{Y}$ structure with a $\mathrm{Zn}$ ion bonded to two fcc $\mathrm{O}$ ions above and an $\mathrm{O}$ ion directly below. The distorted Y structure is marked by the blue dashed ellipse in Fig. 7(e). Lauritsen et al. [7] have earlier proposed a model with only the planar Y structure [shown in Fig. 7(d)]. Their model has ordered regular hexagonal pits, while in our model the pits are composed of rings of 8,10 , and 12 . As the ordering of the three types of rings within a unit cell does not affect much

(b)

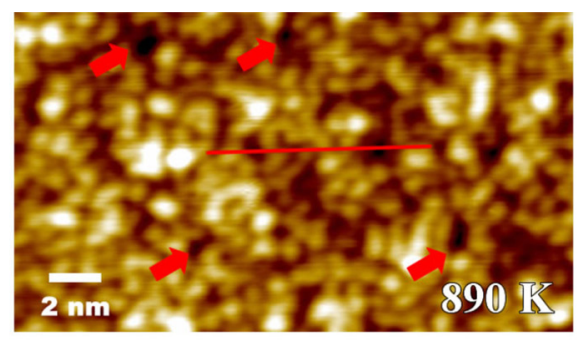

(c)

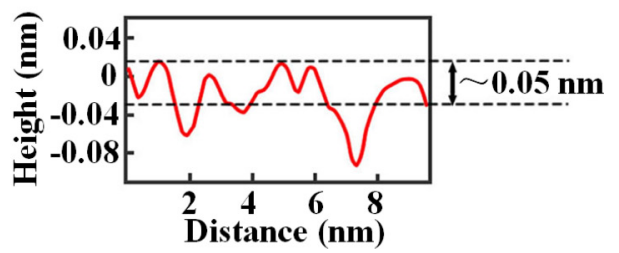

FIG. 6. (Color online) (a) $77 \mathrm{~K}$ STM image of the O-face after annealing at $890 \mathrm{~K}$. (b) Magnified view of the morphology of a terrace like those in (a). (c) Cross-section profile of the line shown in (b). The bias voltage is $+2.0 \mathrm{~V}$ in (a) and $+1.5 \mathrm{~V}$ in (b). 


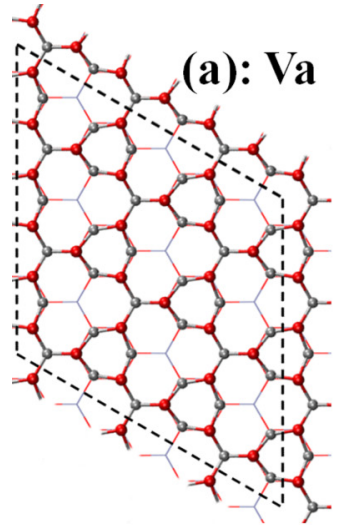

(e): DY

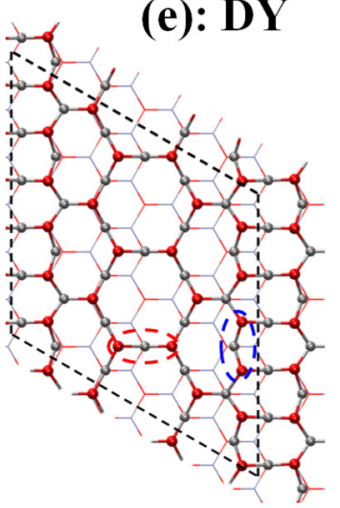

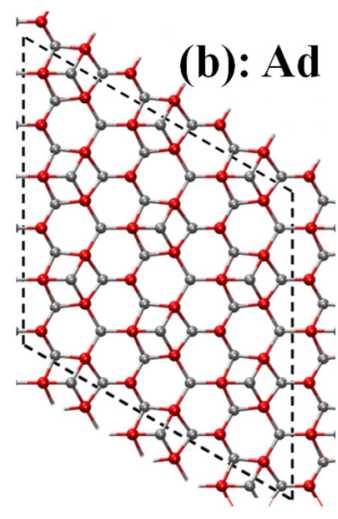

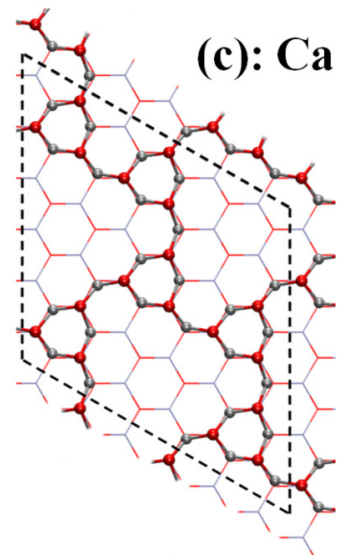

(f)

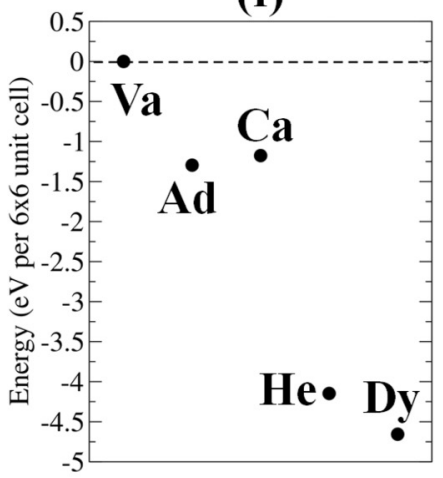

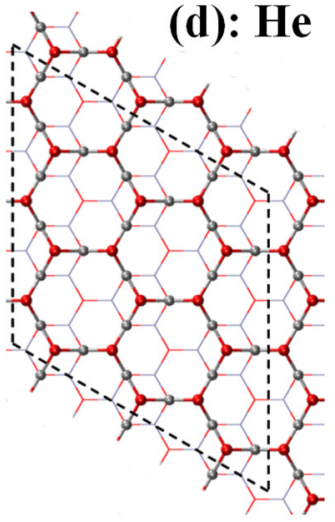

(g)

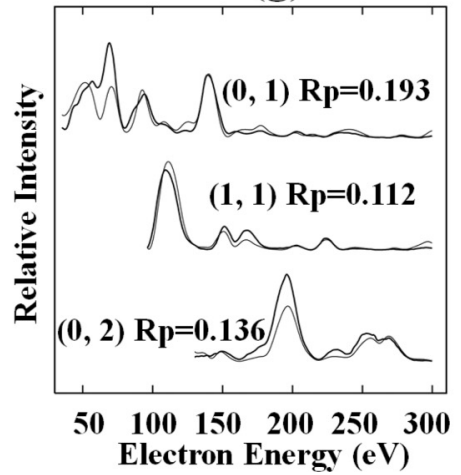

FIG. 7. (Color online) (a)-(e) Top views of reconstructions on the O-face of the first (top) bilayer in a $(6 \times 6)$ unit cell for models: Va, Ad, $\mathrm{Ca}, \mathrm{He}$, and, DY. The boundaries of the unit cell are shown as dashed lines. O: red spheres, Zn: gray spheres. (f) Calculated total energies of the respective models, with the total energy of model (Va) set to zero. (g) Experimental (bold lines) and calculated (light lines) LEED IV curves for the DY model shown in (e). Individual beam $R$-factors are given.

the total energy, we expect that on an extended surface, the pits would not have long range order, a feature in agreement with the STM data. We have used DFT theory to calculate total energies of five reconstructed structures in $(6 \times 6)$ unit cells. The DFT calculations again use seven bilayers, and we stabilize the $\mathrm{Zn}$-face with pseudohydrogen atoms (valence 1.5) placed at on-top sites of a bulklike Zn surface. Structural relaxations are allowed for all atoms in the slab. Referring to Fig. 7, these are (a) $(2 \times 2)$ single $\mathrm{O}$ vacancies $(\mathrm{Va}),(\mathrm{b})(2 \times 2)$ $\mathrm{Zn}$ adatoms (Ad), (c) triangular cavities with $\mathrm{O}$ side $n=3(\mathrm{Ca})$, (d) the ordered hexagonal pit (He) model of Lauritsen et al. [7], and (e) the new DY structure model. As before, we stabilize the $\mathrm{Zn}$-face of the slab with pseudohydrogen atoms (valence 1.5) placed at on-top sites of a bulklike $\mathrm{Zn}$ surface. Structural relaxations are allowed for all atoms in the slab. Models (a), (c), $(\mathrm{d})$, and $(\mathrm{e})$ remove nine net surface $\mathrm{O}$ atoms per $(6 \times 6)$ unit cell (i.e., 25\%), while model (b) adds nine surface $\mathrm{Zn}$ atoms per $(6 \times 6)$ unit cell. The total energies are shown in Fig. 7(f), and the DY structure has the lowest total energy by more than $3.48 \mathrm{eV}$ per $(6 \times 6)$ unit cell compared to the cavity $(\mathrm{Ca})$ model and more than $4.66 \mathrm{eV}$ per $(6 \times 6)$ unit cell compared to the ordered single $\mathrm{O}$ vacancy model (Va). Finally, dynamical LEED analyses carried out for the five models confirm that the DY structure shown in Fig. 7(e) has the lowest Pendry $R$-factor among all models tested. Figure 7(g) shows comparisons of measured and calculated LEED IV curves for the DY structure, which has an overall Pendry $R$-factor of 0.15 .
The two features observed in the STM cross-sectional profile shown in Fig. 6(c) can now be explained vis-a-vis the DY structure of the O-face: The surface roughness between pits of $\sim 0.05 \mathrm{~nm}$ corresponds to a top bilayer thickness, which is compressed from the bulk value [22] because some surface atoms have left the bilayer, leaving a more open bilayer morphology shown in Fig. 7(e). Indeed, the DFT calculated value for the top bilayer of the DY is $0.06 \mathrm{~nm}$, very close to the STM observed corrugation. As for the depth of the pits, the theoretical value is $0.25 \mathrm{~nm}$. This corresponds to the spacing between the top- and second-plane $\mathrm{O}$ ions on the reconstructed O-face. However, due to the small sizes of the pits compared to the size of the STM tip, the measured pit depth is only between $0.01 \mathrm{~nm}-0.07 \mathrm{~nm}$ [e.g., see Fig. 6(c) at $x=2.0 \mathrm{~nm}, 3.9 \mathrm{~nm}$, and $7.4 \mathrm{~nm}$, respectively].

\section{CLEAVAGE ENERGIES OF ZnO POLAR SURFACES}

Now that the reconstruction structures on both polar surfaces are determined, we can calculate the cleavage energy perpendicular to the [0001] direction. The calculations for cleavage energies again use a $(6 \times 6)$ unit cell. Five slabs bounded by reconstruction models are considered. These are as follows: (a) $(2 \times 2)$ ordered single $\mathrm{Zn}$ vacancies on the $\mathrm{Zn}$-face and $(2 \times 2)$ ordered single $\mathrm{O}$ vacancies on the $\mathrm{O}$-face $(\mathrm{Va}+\mathrm{Va}),(\mathrm{b})(2 \times 2)$ ordered $\mathrm{O}$ adatoms on the $\mathrm{Zn}$-face and $(2 \times 2)$ ordered $\mathrm{Zn}$ adatoms on the $\mathrm{O}$-face, both adsorbed at fcc 


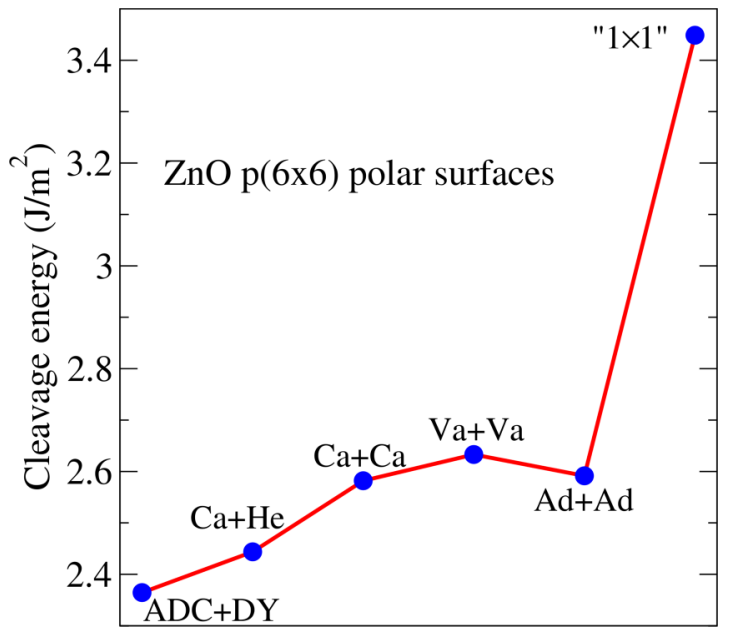

FIG. 8. (Color online) Calculated cleavage energies of six $\mathrm{ZnO}$ slabs; seven bilayers are used for the five slabs with $(6 \times 6)$ reconstructed polar surfaces, and 40 bilayers are used for the slab with relaxed $1 \times 1$ polar surfaces.

hollow sites $(\mathrm{Ad}+\mathrm{Ad}),(\mathrm{c})$ the cavity model [6] with triangular cavities of $\mathrm{Zn}$ side, $n=3$, on the $\mathrm{Zn}$-face and $\mathrm{O}$ side, $n=3$, on the O-face $(\mathrm{Ca}+\mathrm{Ca}),(\mathrm{d})$ the cavity model [6] with triangular cavities of $\mathrm{Zn}$ side, $n=3$, on the $\mathrm{Zn}$-face and the ordered hexagonal Y structure model [7] on the O-face $(\mathrm{Ca}+\mathrm{He})$, and (e) the ADC model on the Zn-face and the DY structure on the O-face $(A D C+D Y)$. Structural coordinates are fully relaxed for all atoms in the slabs. In addition, a 40-bilayer slab bounded on both polar faces by bulklike $(1 \times 1)$ structures

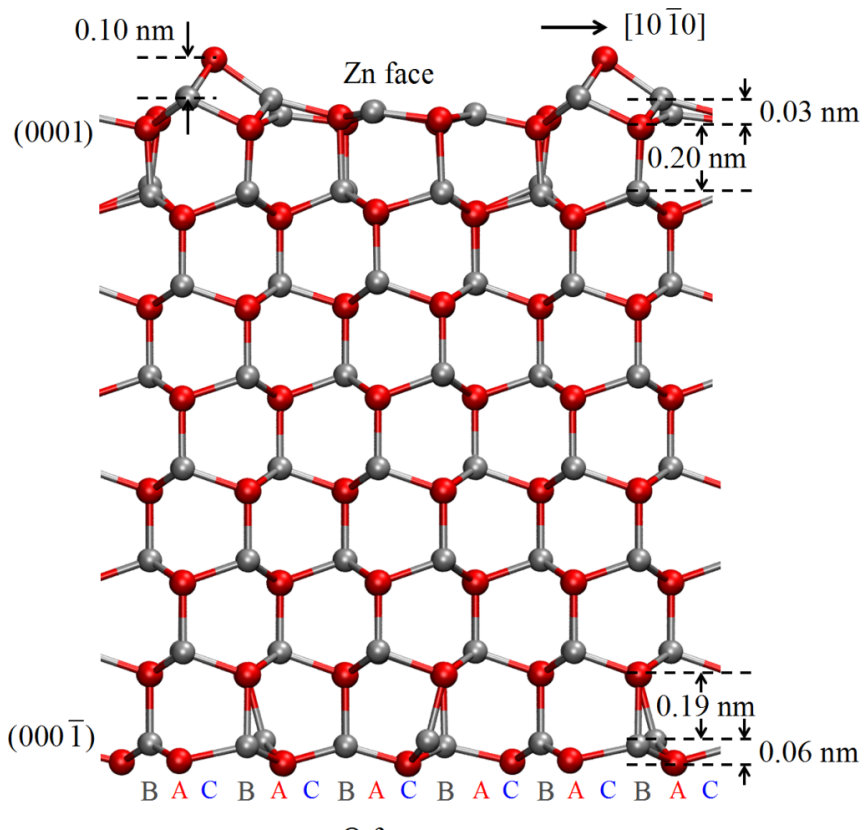

O face

FIG. 9. (Color online) Schematic diagram of a thick $\mathrm{ZnO}$ crystal with a reconstructed $\mathrm{Zn}$-face (top) and $\mathrm{O}$-face (bottom) viewed in the (1120) plane. $\mathrm{O}$ adatoms on the $\mathrm{Zn}$-face and surface $\mathrm{O}$ on the $\mathrm{O}$-face occupy the $\mathrm{C}$ registry (i.e., fcc sites) in the wurtzite crystal. O: red spheres, Zn: gray spheres. Spacings correspond to DFT results. with fully relaxed atomic coordinates is also considered. The cleavage energies of the six slabs are plotted in Fig. 8. The slab bounded by polar surfaces having the reconstruction structures presented in this work, i.e., slab (ADC+DY) has the lowest cleavage energy (i.e., $2.36 \mathrm{~J} / \mathrm{m}^{2}$ ), while the fully relaxed $1 \times 1$ slab has the highest energy (i.e., $3.45 \mathrm{~J} / \mathrm{m}^{2}$ ) by more than $46 \%$. The cleavage energy of $2.36 \mathrm{~J} / \mathrm{m}^{2}$ for the $(\mathrm{ADC}+\mathrm{DY})$ slab is comparable to that of nonpolar surfaces, and this result agrees with the experimental finding [23], which predicted that a significant area of polar surfaces would be exposed in polycrystalline samples. A side view of the $\mathrm{ZnO}$ slab bounded by polar surfaces with the lowest energy reconstruction structures is shown in Fig. 9.

\section{CONCLUSIONS}

The reconstructions on the two polar faces of $\mathrm{ZnO}$ have been studied and the overarching driving force is identified. On both polar faces, there is competition between the electrostatic force and bonding flexibility of undercoordinated $\mathrm{Zn}$ ions, and the latter comes out as the winner. Single vacancies are metastable on the surface, and the electrostatic Madelung force drives to form bigger cavities on both polar surfaces. If the Madelung force dominates, then the formation of very large cavities is preferred on both polar faces (in the Appendix, we show DFT results that indicate that on both the $\mathrm{Zn}$ - and $\mathrm{O}$-faces, single atom vacancy is unstable compared to larger cavities). A competing mechanism is the bonding flexibility of undercoordinated $\mathrm{Zn}$ ions, identified by Lauritsen et al. [7]. This flexibility offers $\mathrm{O}$ atoms low binding energy sites at fcc registries on both surfaces. On the O-face, after some surface $\mathrm{O}$ and $\mathrm{Zn}$ atoms have left lattice sites, part of the remaining $\mathrm{O}$ ions shift from hcp registries to fcc registries to form very energy efficient planar and distorted $\mathrm{Y}$ structures with undercoordinated $\mathrm{Zn}$ ions. The bond configuration of $\mathrm{Zn}$ in the $\mathrm{Y}$ structures is either planar or near planar $\mathrm{sp}^{2}$-type normal to the (0001) plane. Referring to Fig. 7(f), structures containing Y structures (e.g., structures He and DY) are lower in energy by at least $2.85 \mathrm{eV}$ per $(6 \times 6)$ unit cell than structures not containing them (e.g., $\mathrm{Ad}, \mathrm{Ca}$, and $\mathrm{Va}$ ). The resulting surface morphology consists of mainly disordered pits with various shapes, and the surface $\mathrm{O}$ ions occupy both hcp and fcc registries. Similarly, on the $\mathrm{Zn}$-face, O atoms that have left lattice sites when surface cavities are formed rebind on top of three surface $\mathrm{Zn}$ atoms at the fcc registry, resulting in a distorted tetrahedral complex [Fig. 4(d): blue dashed circle] whose energy is much lower than that of the regular threefold bonded $\mathrm{Zn}$ found on the $(1 \times 1)$ unreconstructed surface. This new binding avenue also provides an efficient way to differentially deplete surface $\mathrm{Zn}$ atoms from the $\mathrm{Zn}$-face and diminishes the need for larger cavities to form. Thus, on both polar surfaces, the undercoordinated $\mathrm{Zn}$ ions play a decisive role in shaping the reconstruction structures that cancel the macroscopic dipole moment.

On the $\mathrm{Zn}$-face for samples annealed at $900 \mathrm{~K}$, the very rough terrain inhibits the formation of adatoms: migrating $\mathrm{O}$ atoms are mostly captured at step edges and not as adatoms. This surface is stabilized mainly by cavity formation [6] augmented by some adatoms. As the total energy of the cavity model is over $1.0 \mathrm{eV}$ per $(6 \times 6)$ unit cell higher than that of the 
ADC model [see Fig. 4(e)], the $900 \mathrm{~K}$ surface may be viewed as a metastable structure of the $\mathrm{Zn}$-face, where the most stable structure is only obtained by annealing the surface to higher temperatures, e.g., at $1300 \mathrm{~K}$.

The STM images for both surfaces [e.g., Fig. 2(e) and 6(b)] show different levels of brightness. As the boiling point of $\mathrm{Zn}$ is below the annealing temperatures used to prepare the surfaces, it is unlikely that such brightness levels are caused by structural variations, e.g., Zn droplets on the surface. We rather think that they are caused by variations in the local electric field set up by the disordered distribution of $\mathrm{Zn}$ and $\mathrm{O}$ ions on these polar surfaces.

\section{ACKNOWLEDGMENTS}

This work was supported in part by the Hong Kong Research Grant Council CRF Grant CityU6/CRF/08 and NSFC (Grants No. 11334003, No. 11204185, and No. 11104272). We also thank the National Supercomputing Center in Shenzhen for providing computation time, as well as the High Performance Cluster Computing Centre, Hong Kong Baptist University, which receives funding from the Research Grants Council, University Grants Committee of the HKSAR and Hong Kong Baptist University.
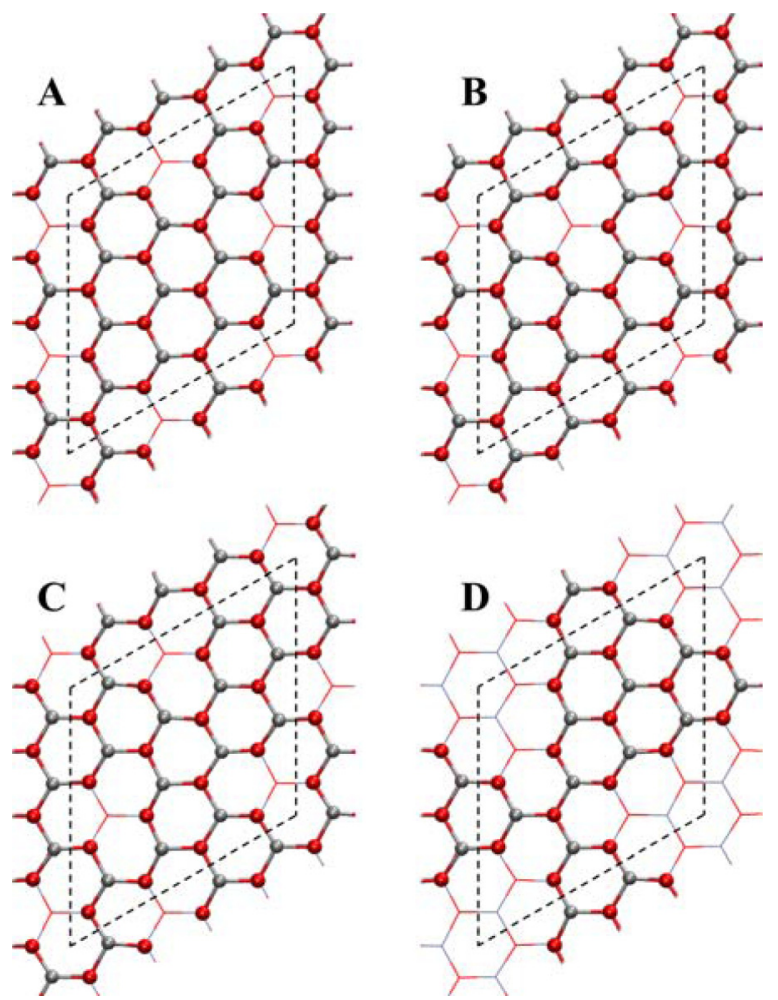

FIG. 10. (Color online) The Zn-face: Structures A-C each contains three single $\mathrm{Zn}$ vacancies per $(4 \times 4)$ unit cell in different arrangements. Structure D contains a triangular cavity with six missing $\mathrm{Zn}$ and three missing $\mathrm{O}$ per $(4 \times 4)$ unit cell. The Madelung energy of structure D containing the cavity has lower energy than that of single vacancies.

\section{APPENDIX: MADELUNG ENERGIES FOR CAVITIES AND SINGLE ATOM VACANCIES}

\section{The Zn-face}

Three equivalent surface $\mathrm{Zn}$ atoms are removed per $(4 \times 4)$ unit cell.

The Madelung energy per $p(4 \times 4)$ unit cell for structure $A$ is set to zero, and the energies of other structures relative to structure A are listed below (see also Fig. 10).

The Madelung energy per $p(4 \times 4)$ unit cell (unit in eV).

\begin{tabular}{lccc}
$\mathrm{A}$ & $\mathrm{B}$ & $\mathrm{C}$ & $\mathrm{D}$ \\
\hline 0.00 & -0.54 & -0.85 & -0.93
\end{tabular}

\section{The O-face}

Three equivalent surface $\mathrm{O}$ atoms are removed per $(4 \times 4)$ unit cell.

The Madelung energy per $p(4 \times 4)$ unit cell for structure $A$ is set to zero, and the energies of other structures relative to structure A are listed below (see also Fig. 11).

The Madelung energy per $p(4 \times 4)$ unit cell (unit in $\mathrm{eV})$.

\begin{tabular}{lccc} 
A & B & C & D \\
\hline 0.00 & -0.34 & -0.39 & -0.60
\end{tabular}
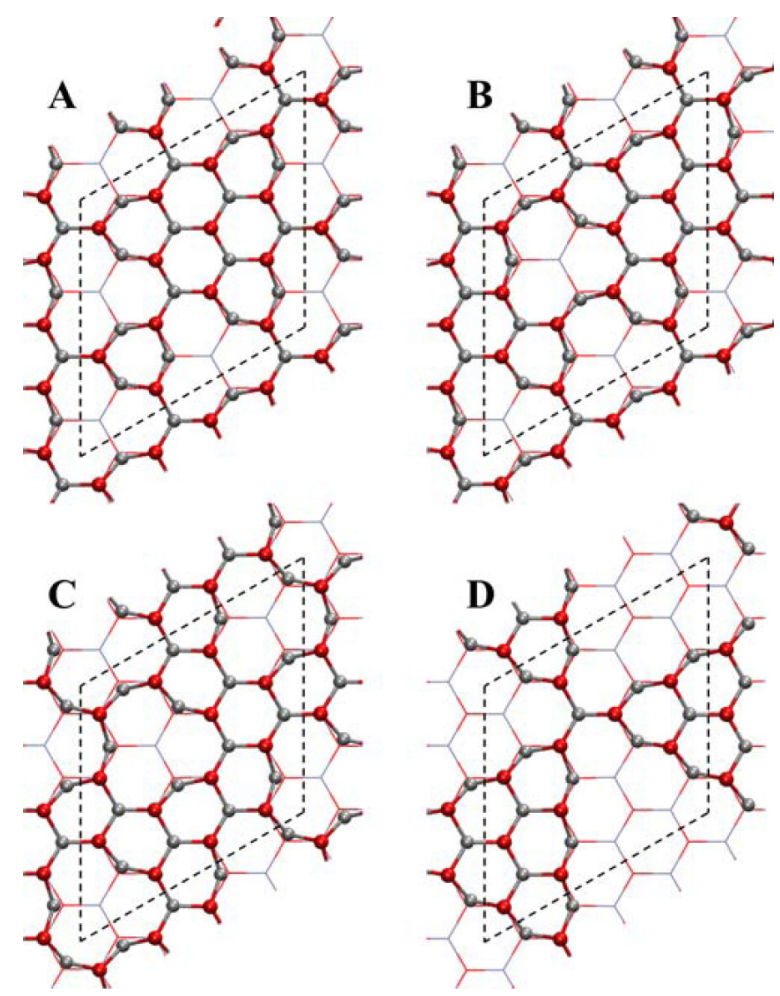

FIG. 11. (Color online) The O-face: Structures A-C each contains three single $O$ vacancies per $(4 \times 4)$ unit cell in different arrangements. Structure D contains a triangular cavity with six missing $\mathrm{O}$ and three missing $\mathrm{Zn}$ per $(4 \times 4)$ unit cell. The Madelung energy of structure D containing the cavity has lower energy than that of single vacancies. 
[1] Z. L. Wang, J. Phys.: Condens. Matter 16, R829 (2004); Z. L. Wang and J. H. Song, Science 312, 242 (2006).

[2] L. Vayssieres, Adv. Mater. 15, 464 (2003).

[3] C. Noguera, J. Phys.: Condens. Matter 12, R367 (2000).

[4] J. Goniakowski, C. Noguera, and L. Giordano, Phys. Rev. Lett. 98, 205701 (2007).

[5] P. W. Tasker, J. Phys. C 12, 4977 (1979).

[6] O. Dulub, U. Diebold, and G. Kresse, Phys. Rev. Lett. 90, 016102 (2003).

[7] J. Lauritsen, S. Porsgaard, M. K. Rasmussen, M. C. R. Jensen, R. Bechstein, K. Meinander, B. S. Clausen, S. Helveg, R. Wahl, G. Kresse, and F. Besenbacher, ACS Nano 5, 5987 (2011).

[8] G. Kresse, O. Dulub, and U. Diebold, Phys. Rev. B 68, 245409 (2003).

[9] G. Kresse and J. Furthmüller, Phys. Rev. B 54, 11169 (1996).

[10] P. E. Blöchl, Phys. Rev. B 50, 17953 (1994).

[11] J. P. Perdew and Y. Wang, Phys. Rev. B 45, 13244 (1992).

[12] G. Henkelman, B. P. Uberuaga, and H. Jónsson, J. Chem. Phys. 113, 9901 (2000).

[13] M. A. Van Hove, W. Moritz, H. Over, P. J. Rous, A. Wander, A. Barbieri, N. Materer, U. Starke, and G. A. Somorjai, Surf. Sci. Rep. 19, 191 (1993).

[14] A. Barbieri and M. A. Van Hove, software available at http://www.icts.hkbu.edu.hk/vanhove/VanHove_files/leed/ leedpack.html

[15] J. B. Pendry, J. Phys. C 13, 937 (1980).

[16] M. H. Xie, S. M. Seutter, W. K. Zhu, L. X. Zheng, H. S. Wu, and S. Y. Tong, Phys. Rev. Lett. 82, 2749 (1999).

[17] K. Heinz, U. Starke, M. A. Van Hove, and G. A. Somorjai, Surf. Sci. 261, 57 (1992).

[18] D. K. Saldin, X. Chen, J. A. Vamvakas, M. Ott, H. Wedler, K. Reuter, K. Heiz, and P. L. De Andres, Surf. Rev. Lett. 4, 991 (1997).

[19] C. Wöll, Prog. Surf. Sci. 82, 55 (2007).

[20] M. Kunat, St. Gil Girol, Th. Becker, Ch. Burghaus, and C. Wöll, Phys. Rev. B 66, 081402 (2002).

[21] H. S. Qiu, B. Meyer, Y. M. Wang, and C. Wöll, Phys. Rev. Lett. 101, 236401 (2008).

[22] S. E. Chamberlin, C. J. Hirschmugl, S. T. King, H. C. Poon, and D. K. Saldin, Phys. Rev. B 84, 075437 (2011).

[23] M. Bowker, H. Houghton, K. C. Waugh, T. Giddings, and M. Green, J. Catal. 84, 252 (1983). 